Tropical Journal of Pharmaceutical Research January 2015; 14 (1): 47-54

ISSN: $1596-5996$ (print); 1596-9827 (electronic)

(C) Pharmacotherapy Group, Faculty of Pharmacy, University of Benin, Benin City, 300001 Nigeria.

All rights reserved.

Available online at http://www.tjpr.org

Original Research Article

http://dx.doi.org/10.4314/tjpr.v14i1.8

\title{
Evaluation of Sulfonamide Derivatives of Dagenan Chloride as Lipoxygenase and $\alpha-G$ lucosidase Inhibitors
}

\author{
Muhammad A Abbasi ${ }^{1 *}$, Saima Najm ${ }^{1}$, Aziz-ur-Rehman ${ }^{1}$, Shahid Rasool ${ }^{1}$, Khalid $^{3}$ \\ M Khan ${ }^{2}$, Muhammad Ashraf ${ }^{3}$, Rumana Nasar ${ }^{3}$ and Umber Alam ${ }^{3}$ \\ ${ }^{1}$ Department of Chemistry, Government College University, Lahore-54000, ${ }^{2} \mathrm{HEJ}$ Research Institute of Chemistry, International \\ Center for Chemical and Biological Sciences, University of Karachi, Karachi-75270, ${ }^{3}$ Department of Biochemistry and \\ Biotechnology, The Islamia University of Bahawalpur, Bahawalpur-63100, Pakistan
}

${ }^{*}$ For correspondence: Email: atrabbasi@yahoo.com; Tel: (+92)-42-111000010 ext.266

Received: 6 January 2014

Revised accepted: 14 November 2014

\begin{abstract}
Purpose: To study the enzyme inhibition activity of various sulfonamides derived from dagenan chloride.

Methods: The synthesis of $\mathrm{N}$-(naphthalen-1-yl)-4-acetamidobenzenesulfonamide (3) was carried out by gearing up 1-naphthylamine (1) with dagenan chloride (2) in water in the presence of $\mathrm{Na}_{2} \mathrm{CO}_{3}$ solution. Further, compound 3 was treated with various alkyl/aralkyl halides (4a-o) to yield $5 a-0$ in an aprotic polar solvent, DMF (dimethylformamide), using $\mathrm{LiH}$ as activator. The structures of all the synthesized molecules were corroborated by infra red spectroscopy (IR), proton nuclear magnetic resonance $(1 \mathrm{H}$ $N M R)$ and electron impact mass spectrometry (EI-MS) and screened against lipoxygenase and $\alpha$ glucosidase using baicalein and acarbose as reference standards, respectively.

Results: Molecules $5 e$ and $5 j$ showed good lipoxygenase inhibition activity with $I C_{50}(50 \%$ inhibition concentration) value of $132.21 \pm 0.73$ and $133.33 \pm 0.87 \mu \mathrm{mol} / \mathrm{L}$, respectively, relative to reference, while $5 \mathrm{~m}$ was the most active inhibitor of $\alpha$-glucosidase with $I C_{50}$ of $19.41 \pm 0.55 \mu \mathrm{mol} / \mathrm{L}$ relative to reference.

Conclusion: Most of the synthesized compounds are good inhibitors of lipoxygenase but moderate inhibitors of a-glucosidase enzyme. These molecules should be evaluated for their in vivo activity to determine their potentials as anti-inflammatory and anti-diabetic drugs.
\end{abstract}

Keywords: 1-Naphthylamine, Sulfonamide, Lipoxygenase, a-Glucosidase, Dagenan chloride, Enzyme inhibitor, Anti-inflammatory, Anti-diabetic

\footnotetext{
Tropical Journal of Pharmaceutical Research is indexed by Science Citation Index (SciSearch), Scopus, International Pharmaceutical Abstract, Chemical Abstracts, Embase, Index Copernicus, EBSCO, African Index Medicus, JournalSeek, Journal Citation Reports/Science Edition, Directory of Open Access Journals (DOAJ), African Journal Online, Bioline International, Open-J-Gate and Pharmacy Abstracts
}

\section{INTRODUCTION}

Sulfonamides have been used as therapeutic agent, first as antibacterial agents but later extended to treat other diseases. The first sulfonamide which was identified as the active in vivo metabolite of red azo dye was prontosil [13]. In addition, sulfonamides are known to inhibit various enzymes such as carbonic anhydrase [4]. Sulfonamides due to their resemblance to $p$ - aminobenzoic acid (PABA), act as competitive inhibitors and so block the synthesis of tetrahydrofolic acid required by bacteria for growth $[3,5]$.

Lipoxygenase (EC 1.13.11.12) extensively exists in animals and plants. Iron $\left(\mathrm{Fe}^{2+}\right)$ structurally present in lipoxygenase gets oxidized to catalytically active $\mathrm{Fe}^{+3}$ products including hydro peroxide derivatives such as 15-hydroperoxy- 
eicosatetraenoic acid (15-HPETE), leukotrienes from arachidonic acid as a substrate, and 13hydroperoxy-octadecadienoic acid (13-HPODE) from linoleic acid as a substrate [6]. One of the key products of lipoxygenases consists of bioactive lipids known as leukotrienes [7]. Leukotrienes are potential mediators of various inflammatory disorders such as bronchial asthma [8]. a-Glucosidase (EC 3.2.1.20) is located in the brush-border surface membrane of small intestinal cells [9]. $\alpha$-Glucosidase inhibitors are used as oral anti-diabetic drugs for patients with type-2 diabetic mellitus. Postprandial hyperglycemia has a vital role in the development of type-2 diabetes and complications associated with this disease such as nephropathy, neuropathy, micoangiopathy and macroangipathy [10]. The inhibitors of this enzyme can retard the liberation of D-glucose of oligosaccharides and disaccharides from dietary complex carbohydrates and delay glucose absorption, resulting in reduced postprandial hyperglycemia [11].

Literature survey has revealed that even minor modifications in the structure of molecules can be responsible for varied anti-microbial activities. In continuation of our previous work in this regard [12-14], new sulfonamides derived from dagenan chloride have been synthesized in order to assess their enzyme inhibition activities.

\section{EXPERIMENTAL}

Thin layer chromatography (TLC) was performed on pre-coated silica gel G-25-UV254 plates using ethyl acetate and $n$-hexane solvent systems, with detection under $254 \mathrm{~nm} .{ }^{1} \mathrm{H}-\mathrm{NMR}$ spectra, with $\delta$ values in ppm and $\mathrm{J}$-values in $\mathrm{Hz}$, were recorded in $\mathrm{CDCl}_{3}$ using Bruker spectrometer at $400 \mathrm{MHz}$. IR spectra was recorded by $\mathrm{KBr}$ pellet method using Jasco-320-A spectrophotometer. Mass spectra was measured on Finnigan MAT-112 instrument and melting point on Gallonkamp apparatus by open capillary tube and was uncorrected.

\section{Procedure for synthesis of $\mathrm{N}$-(naphthalen-1-} yl)-4-acetamidobenzenesulfonamide (3)

An equimolar mixture of 1-naphthylamine (1; $10.0 \mathrm{mmol}$ ) and dagenan chloride $(2 ; 10.0 \mathrm{mmol})$ was suspended in $25 \mathrm{~mL}$ water, in a round bottom (RB) flask (100 ml), with $\mathrm{pH}=9$, adjusted and maintained by aqueous $\mathrm{Na}_{2} \mathrm{CO}_{3}$ at room temperature. The reaction solution was stirred for $2 \mathrm{~h}$ and monitored by TLC. Concentrated $\mathrm{HCl}$ was added gradually to adjust the $\mathrm{pH}$ to 2.0 . The precipitate was collected by filtration, washed with distilled water and dried to afford compound 3. The product was re-crystallized from methanol.

General procedure for synthesis of $\mathrm{N}$ alkyl/aralkyl-N-(naphthalen-1-yl)-4acetamidobenzenesulfonamide (5a-o)

Molecule $3(0.1 \mathrm{mmol})$ was taken in a RB flask $(50 \mathrm{ml})$ and dissolved in DMF $(10.0 \mathrm{ml})$, followed by the addition of $\mathrm{LiH}(0.1 \mathrm{mmol})$. The mixture was stirred for $30 \mathrm{~min}$ at room temperature and then alkyl/aralkyl halides (4a-o) were added. Further stirring was continued for $3 \mathrm{~h}$ along with TLC monitoring. The products, 5a-o, were precipitated by adding water and were isolated by filtration, washing with distilled water; and crystallized from methanol.

\section{Lipoxygenase assay}

Lipoxygenase activity was assayed by a previously reported method [15] with slight modifications. Lipoxygenase assay mixture was prepared with sodium phosphate buffers, test compound and enzyme. After thoroughly mixing, pre-reading and pre-incubating, substrate was added. The variation in absorbance was noted after incubation. The experiments were performed in triplicate with baicalein as reference.

\section{a-Glucosidase assay}

a-Glucosidase inhibition activity was performed following a slightly modified method [11]. Like lipoxygenase assay, a-glucosidase assay was carried out using phosphate buffer saline, test compound and enzyme. After mixing, preincubation and pre-reading, a substrate ( $p$ nitrophenyl glucopyranoside) was added for initiation. The absorbance was noted after incubation. The experiments were performed in triplicate with acarbose as reference.

\section{Statistical analysis}

Determinations were carried out in triplicate and statistical analysis was performed using Microsoft Excel 2010, and the results presented as mean $\pm \mathrm{SEM}$. $I \mathrm{C}_{50}$ values (concentration at which there is $50 \%$ in enzyme catalyzed reaction) compounds were calculated using EZFit Enzyme Kinetics Software (Perrella Scientific Inc. Amherst, USA). $I C_{50}$ values were calculated (as mean of three independent experiments) from the graph by dilution of compounds to different concentrations. The statistical analysis included $85 \% \mathrm{CL}$. 
<smiles>Nc1cccc2ccccc12</smiles>

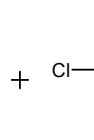<smiles>CC(=O)Nc1ccc(S(=O)(=O)Cl)cc1</smiles><smiles>CC(=O)Nc1ccc(S(=O)(=O)Nc2cccc3ccccc23)cc1</smiles>

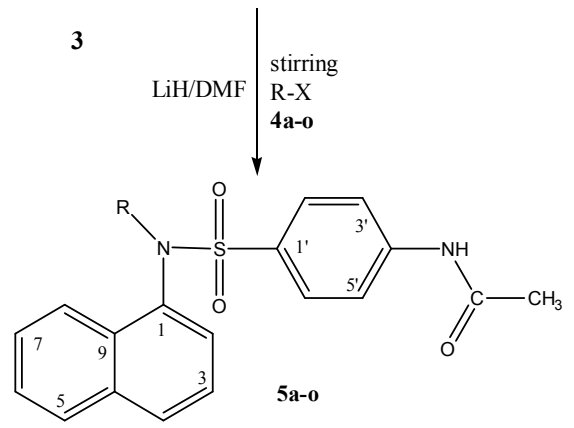

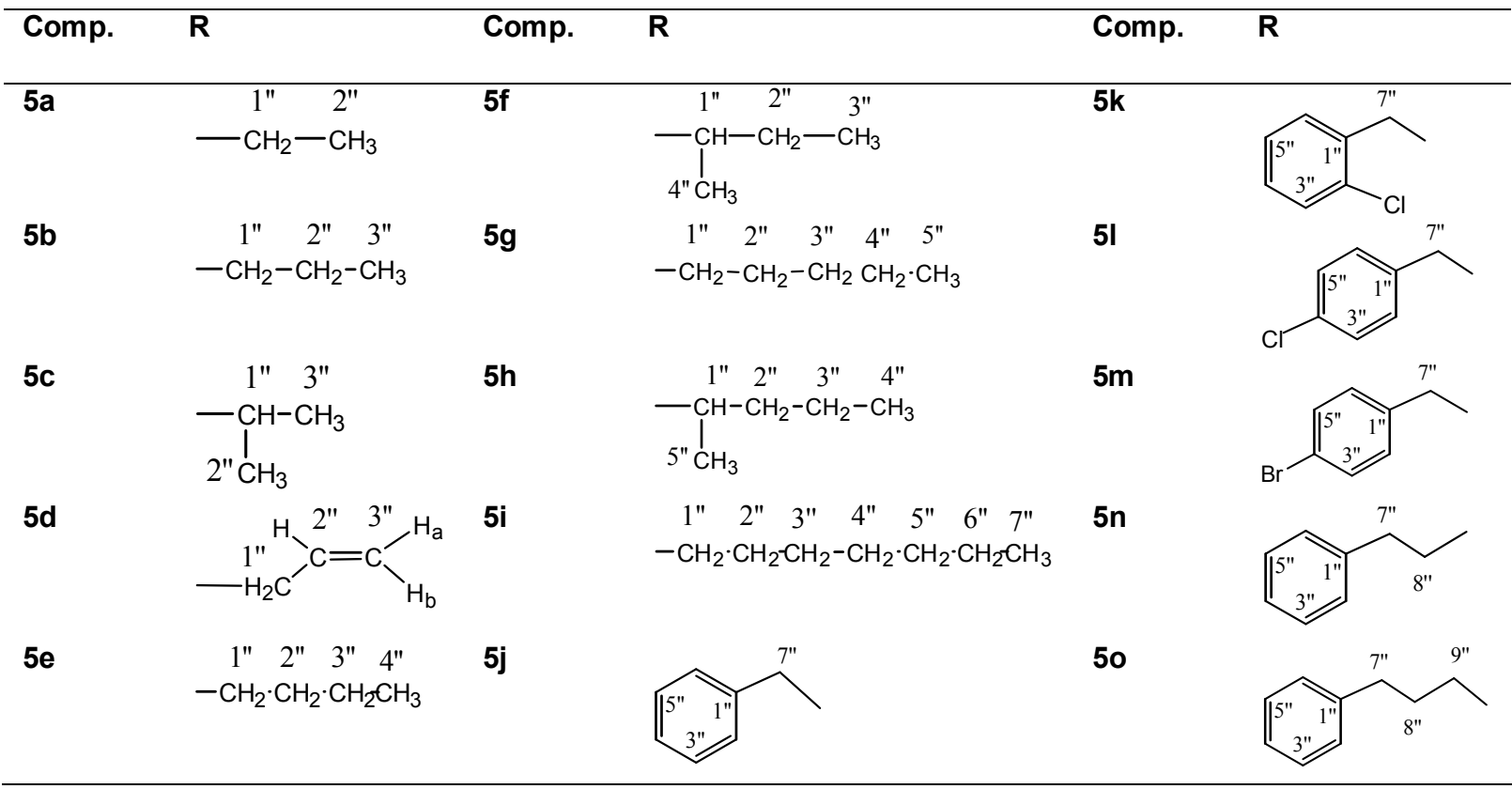

Scheme 1: $\mathrm{N}$-alkyl/aralkly substituted derivatives of dagenan chloride (2)

\section{RESULTS}

The spectral data for the synthesized molecules $(3,5 a-0)$ are as follows.

\section{$N$-(Naphthalen-1-yl)-4- acetamidobenzenesulfonamide (3)}

Move Amorphous Solid; Yield: 80 \%; M.P: 222 ${ }^{\circ} \mathrm{C}$; Molecular Formula: $\mathrm{C}_{18} \mathrm{H}_{16} \mathrm{~N}_{2} \mathrm{O}_{3} \mathrm{~S}$; Molecular Mass: 340 ; IR $\left(\mathrm{KBr}, \mathrm{cm}^{-1}\right) v_{\max }: 3500(\mathrm{~N}-\mathrm{H}), 3080$ (Ar C-H), 1650 (Ar C=C), $1645(-\mathrm{HNC}=\mathrm{O}), 1390$ $\left(\mathrm{SO}_{2}-\right) ;{ }^{1} \mathrm{H}-\mathrm{NMR}\left(\mathrm{CDCl}_{3}, 400 \mathrm{MHz}, \delta / \mathrm{ppm}\right): 8.24$ (d, $\left.J=8.4 \mathrm{~Hz}, 2 \mathrm{H}, \mathrm{H}-3^{\prime} \& \mathrm{H}-5^{\prime}\right), 7.80$ (d, $J=6.4$ $\mathrm{Hz}, 1 \mathrm{H}, \mathrm{H}-4), 7.66$ (dd, $J=6.4,1.9 \mathrm{~Hz}, 1 \mathrm{H}, \mathrm{H}-5$ ), $7.66(\mathrm{dd}, J=6.4,1.9 \mathrm{~Hz}, 1 \mathrm{H}, \mathrm{H}-8), 7.49$ (dt, $J=$ 8.4, 3.6 Hz, 1H, H-6), $7.49(\mathrm{dt}, J=8.4,3.6 \mathrm{~Hz}$, $1 \mathrm{H}, \mathrm{H}-7), 7.43(\mathrm{t}, J=8.0 \mathrm{~Hz}, 1 \mathrm{H}, \mathrm{H}-3), 7.35(\mathrm{~d}, J$
$=6.4 \mathrm{~Hz}, 1 \mathrm{H}, \mathrm{H}-2), 6.66\left(\mathrm{~d}, J=8.4 \mathrm{~Hz}, 2 \mathrm{H}, \mathrm{H}-2^{\prime}\right.$ \& H-6'), $2.16\left(\mathrm{~s}, 3 \mathrm{H},-\mathrm{COCH}_{3}\right)$; El-MS $(\mathrm{m} / \mathrm{z}): 340$ (3\%), 275 (17\%), $198(7 \%), 134(10 \%), 127$ (100\%), $102(2 \%), 65(34 \%), 51(10 \%)$.

\section{$\mathrm{N}$-Ethyl-N-(naphthalen-1-yl)-4- acetamidobenzenesulfonamide (5a)}

Move Amorphous Solid; Yield: 70 \%; M.P: 186 ${ }^{\circ} \mathrm{C}$; Molecular Formula: $\mathrm{C}_{20} \mathrm{H}_{25} \mathrm{~N}_{2} \mathrm{O}_{3} \mathrm{~S}$; Molecular Mass: 368; IR ( $\left.\mathrm{KBr}, \mathrm{cm}^{-1}\right) v_{\text {max }} 3080(\mathrm{Ar} \mathrm{C}-\mathrm{H})$, 1650 ( $\mathrm{Ar} \mathrm{C}=\mathrm{C}), 1645(-\mathrm{HNC}=\mathrm{O}), 1390\left(\mathrm{SO}_{2^{-}}\right)$; ${ }^{1} \mathrm{H}-\mathrm{NMR}\left(\mathrm{CDCl}_{3}, 400 \mathrm{MHz}, \delta / \mathrm{ppm}\right): 7.97$ (d, $J=$ $7.9 \mathrm{~Hz}, 2 \mathrm{H}, \mathrm{H}-3^{\prime}$ \& H-5'), 7.84 (dd, $J=8.0,4.2$ $\mathrm{Hz}, 1 \mathrm{H}, \mathrm{H}-5), 7.82$ (dd, $J=8.0,3.4 \mathrm{~Hz}, 1 \mathrm{H}, \mathrm{H}-8$ ), 7.75 (d, $J=7.9 \mathrm{~Hz}, 1 \mathrm{H}, \mathrm{H}-4), 7.67$ (t, $J=11.2 \mathrm{~Hz}$, $1 \mathrm{H}, \mathrm{H}-3), 7.50(\mathrm{~d}, J=6.0 \mathrm{~Hz}, 1 \mathrm{H}, \mathrm{H}-2), 7.38(\mathrm{t}, J$ $=7.9 \mathrm{~Hz}, 1 \mathrm{H}, \mathrm{H}-6), 7.38(\mathrm{dt}, J=8.0,7.9 \mathrm{~Hz}, 1 \mathrm{H}$, 
$\mathrm{H}-7), 7.05$ (d, J = 7.9 Hz, 2H, H-2' \& H-6'), 3.23 (q, J = 7.2 Hz, 2H, H-1"), 3.76 (t, J = 6.7 Hz, $3 \mathrm{H}$, $\mathrm{CH}_{3}-2$ "), 2.21 (s, 3H, $\left.-\mathrm{COCH}_{3}\right)$; El-MS (m/z): 368 (9\%), 275 (13\%), 198 (5\%), 134 (9\%), 127 (100\%), 102 (4\%), 65 (43\%), 51 (5\%), 29 (5 $\%)$.

\section{N-(Propan-1-yl)-N-(naphthalen-1-yl)-4- acetamidobenzenesulfonamide (5b)}

Black Sticky Solid; Yield: $65 \%$; Molecular Formula: $\mathrm{C}_{21} \mathrm{H}_{22} \mathrm{~N}_{2} \mathrm{O}_{3} \mathrm{~S}$; Molecular Mass: 382; IR $\left(\mathrm{KBr}, \mathrm{cm}^{-1}\right) v_{\text {max }}: 3650$ (Ar C-H), 1660 (Ar C=C), 1655 (-HNC=O), $1400\left(\mathrm{SO}_{2-}\right) ;{ }^{1} \mathrm{H}-\mathrm{NMR}\left(\mathrm{CDCl}_{3}\right.$, $400 \mathrm{MHz}, \delta / \mathrm{ppm}): 8.20$ (dd, $J=7.2,1.6 \mathrm{~Hz}, 1.6$ $\mathrm{Hz}, 1 \mathrm{H}, \mathrm{H}-8), 7.83-7.78$ (m, 2H, H-5 \& H-6), 7.72 (d, J = 8.4 Hz, 2H, H-3' \& H-5'), 7.59 (d, J = 8.0 $\mathrm{Hz}, 1 \mathrm{H}, \mathrm{H}-7), 7.32$ (t, J = 7.6 Hz, 1H, H-3), 7.24 $(\mathrm{d}, J=8.4 \mathrm{~Hz}, 1 \mathrm{H}, \mathrm{H}-4), 7.05(\mathrm{~d}, J=6.8 \mathrm{~Hz}, 1 \mathrm{H}$, H-2), 6.81 (d, J = 7.2 Hz, 2H, H-2' \& H-6'), 3.67 (t, $J=7.6 \mathrm{~Hz}, 2 \mathrm{H}, \mathrm{H}-1 "), 2.16\left(\mathrm{~s}, 3 \mathrm{H},-\mathrm{COCH}_{3}\right)$, 1.52-1.50 (m, 2H, H-2"), $0.83(\mathrm{t}, J=7.6 \mathrm{~Hz}, 3 \mathrm{H}$, $\mathrm{CH}_{3}-3$ "); El-MS (m/z): 382 (7\%), 275 (6\%), 198 (13\%), 134 (15\%), 127 (100\%), 102 (3\%), 65 (41\%), 51 (3\%), 43 (4\%).

\section{N-(Propan-2-yl)-N-(naphthalen-1-yl)-4- acetamidobenzenesulfonamide (5c)}

Pink Crystalline Solid; Yield: 70 \%; M.P: $225{ }^{\circ} \mathrm{C}$; Molecular Formula: $\mathrm{C}_{21} \mathrm{H}_{22} \mathrm{~N}_{2} \mathrm{O}_{3} \mathrm{~S}$; Molecular Mass: 382 ; IR $\left(\mathrm{KBr}, \mathrm{cm}^{-1}\right) v_{\max }: 3018(\mathrm{Ar} \mathrm{C}-\mathrm{H})$, 1678 ( $\mathrm{Ar} \mathrm{C}=\mathrm{C}), 1657$ (-HNC=O), $1396\left(\mathrm{SO}_{2^{-}}\right)$; ${ }^{1} \mathrm{H}-\mathrm{NMR}\left(\mathrm{CDCl}_{3}, 400 \mathrm{MHz}, \delta / \mathrm{ppm}\right): 8.15(\mathrm{~d}, \mathrm{~J}=$ $8.4 \mathrm{~Hz}, 1 \mathrm{H}, \mathrm{H}-8), 7.92-7.82(\mathrm{~m}, 2 \mathrm{H}, \mathrm{H}-5$ \& $\mathrm{H}-6)$, $7.72\left(\mathrm{~d}, J=6.4 \mathrm{~Hz}, 2 \mathrm{H}, \mathrm{H}-3^{\prime}\right.$ \& H-5') 7.71 (d, J = $6.4 \mathrm{~Hz}, 1 \mathrm{H}, \mathrm{H}-4), 7.63(\mathrm{t}, J=5.6 \mathrm{~Hz}, 1 \mathrm{H}, \mathrm{H}-3)$, $7.57(\mathrm{~d}, J=8.8 \mathrm{~Hz}, 1 \mathrm{H}, \mathrm{H}-2), 7.50$ (d, $J=8.8 \mathrm{~Hz}$, $\left.2 \mathrm{H}, \mathrm{H}-22^{\prime} \& \mathrm{H}-6{ }^{\prime}\right), 7.40-7.31(\mathrm{~m}, 1 \mathrm{H}, \mathrm{H}-7), 3.31-$ $3.30(\mathrm{~m}, 1 \mathrm{H}, \mathrm{H}-1 "), 2.05\left(\mathrm{~s}, 3 \mathrm{H},-\mathrm{COCH}_{3}\right), 0.93$ (d, J = 6.8 Hz, 6H, $\left.\mathrm{CH}_{3}-2 ", \mathrm{CH}_{3}-3 "\right)$; El-MS (m/z): 382 (5 \%), 275 (9\%), 198 (23\%), 134 (19\%), $127(100 \%), 102(1 \%), 65$ (37\%), 51 (6\%), 43 (2\%).

\section{$\mathbf{N}$-Allyl-N-(naphthalen-1-yl)-4- acetamidobenzenesulfonamide (5d)}

Move Amorphous Solid; Yield: 80 \%; M.P: 177 ${ }^{\circ} \mathrm{C}$; Molecular Formula: $\mathrm{C}_{21} \mathrm{H}_{20} \mathrm{~N}_{2} \mathrm{O}_{3} \mathrm{~S}$; Molecular Mass: 380; IR ( $\left.\mathrm{KBr}, \mathrm{cm}^{-1}\right)$ vmax: 3082 (Ar C-H), 1679 ( $\mathrm{Ar} \mathrm{C}=\mathrm{C}), 1647$ (-HNC=O), $1398\left(\mathrm{SO}_{2-}\right)$; $1 \mathrm{H}-\mathrm{NMR}\left(\mathrm{CDCl}_{3}, 400 \mathrm{MHz}, \delta / \mathrm{ppm}\right): 8.15(\mathrm{~d}, J=$ $8.0 \mathrm{~Hz}, 2 \mathrm{H}, \mathrm{H}-3^{\prime}$ \& H-5'), $7.82(\mathrm{t}, J=8.4 \mathrm{~Hz}, 1 \mathrm{H}$, $\mathrm{H}-8), 7.67-7.62$ (m, 2H, H-5 \& H-6), 7.51-7.48 (m, $2 \mathrm{H}, \mathrm{H}-4$ \& $\mathrm{H}-7), 7.31(\mathrm{t}, J=8.0 \mathrm{~Hz}, 1 \mathrm{H}, \mathrm{H}-3)$, $7.00\left(\mathrm{~d}, J=7.2 \mathrm{~Hz}, 2 \mathrm{H}, \mathrm{H}-2 ' \& \mathrm{H}-66^{\prime}\right), 6.88$ (d, $J=$ $7.2 \mathrm{~Hz}, 1 \mathrm{H}, \mathrm{H}-2), 5.75-5.73$ (m, 1H, H-2"), 4.95 (dd, $\left.J=7.6,3.2 \mathrm{~Hz}, 1 \mathrm{H}, \mathrm{H}_{\mathrm{a}}-3^{\prime \prime}\right), 4.16$ (dd, $J=7.2$, $\left.3.6 \mathrm{~Hz}, 1 \mathrm{H}, \mathrm{H}_{\mathrm{b}}-3^{\prime \prime}\right), 2.87$ (s, 2H, H-1"), 2.20 (s,
3H, -COCH ${ }_{3}$; El-MS (m/z): 380 (9\%), 275 (15 \%), 198 (21\%), 134 (17\%), 127 (100\%), 102 (4 $\%), 65(47 \%), 51(4 \%), 41(3 \%)$.

\section{N-(Butan-1-yl)-N-(naphthalen-1-yl)-4- acetamidobenzenesulfonamide (5e)}

Black Sticky Solid; Yield: $65 \%$; Molecular Formula: $\mathrm{C}_{22} \mathrm{H}_{24} \mathrm{~N}_{2} \mathrm{O}_{3} \mathrm{~S}$; Molecular Mass: 396; IR $\left(\mathrm{KBr}, \mathrm{cm}^{-1}\right) v_{\text {max }}: 3015(\mathrm{Ar} \mathrm{C}-\mathrm{H}), 1689$ (Ar C=C), 1657 (-HNC=O), $1396\left(\mathrm{SO}_{2}-\right) ;{ }^{1} \mathrm{H}-\mathrm{NMR}\left(\mathrm{CDCl}_{3}\right.$, $400 \mathrm{MHz}, \delta / \mathrm{ppm}): 8.15$ (d, J = $7.2 \mathrm{~Hz}, 1 \mathrm{H}, \mathrm{H}-8)$, $7.94\left(\mathrm{~d}, J=8.0 \mathrm{~Hz}, 2 \mathrm{H}, \mathrm{H}-3^{\prime} \& \mathrm{H}-5^{\prime}\right), 7.83$ (d, $J=$ $8.0 \mathrm{~Hz}, 1 \mathrm{H}, \mathrm{H}-5), 7.72$ (d, J = $8.4 \mathrm{~Hz}, 1 \mathrm{H}, \mathrm{H}-4)$, $7.62(\mathrm{~d}, J=5.6 \mathrm{~Hz}, 1 \mathrm{H}, \mathrm{H}-6), 7.51(\mathrm{dt}, J=6.0$, $3.2 \mathrm{~Hz}, 1 \mathrm{H}, \mathrm{H}-7), 7.37$ (t, $J=8.0 \mathrm{~Hz}, 1 \mathrm{H}, \mathrm{H}-3)$, 7.05 (d, J = 7.2 Hz, 2H, H-2' \& H-6'), 6.87 (d, J = $7.2 \mathrm{~Hz}, 1 \mathrm{H}, \mathrm{H}-2), 3.71$ (t, $J=7.6 \mathrm{~Hz}, 2 \mathrm{H}, \mathrm{H}-1 ")$, $2.20\left(\mathrm{~s}, 3 \mathrm{H},-\mathrm{COCH}_{3}\right), 1.55-1.35(\mathrm{~m}, 2 \mathrm{H}, \mathrm{H}-2 ")$, $0.89\left(\mathrm{t}, J=7.2 \mathrm{~Hz}, 3 \mathrm{H}, \mathrm{CH}_{3}-4 "\right), 1.32-1.22(\mathrm{~m}$, 2H, $\left.\mathrm{CH}_{2}-3 "\right)$; El-MS (m/z): 396 (4\%), 275 (5\%), 198 (27\%), 134 (18\%), 127 (100\%), 102 (3\%), 65 (39\%), 57 (6 \%), 51 (4\%).

\section{N-(Butan-2-yl)-N-(naphthalen-1-yl)-4- acetamidobenzenesulfonamide (5f)}

Purple Amorphous Solid; Yield: 80 \%; M.P: 183 ${ }^{\circ} \mathrm{C}$; Molecular Formula: $\mathrm{C}_{22} \mathrm{H}_{24} \mathrm{~N}_{2} \mathrm{O}_{3} \mathrm{~S}$; Molecular Mass: 396 ; IR $\left(\mathrm{KBr}, \mathrm{cm}^{-1}\right) v_{\max }: 3053(\mathrm{Ar} \mathrm{C}-\mathrm{H})$, 1679 (-HNC=O), $\left.1592(\mathrm{C}=\mathrm{C}), 1398\left(\mathrm{SO}_{2}\right)^{-}\right) ;{ }^{1} \mathrm{H}-$ NMR $\left(\mathrm{CDCl}_{3}, 400 \mathrm{MHz}, \delta / \mathrm{ppm}\right): 8.29(\mathrm{~d}, J=8.0$ $\mathrm{Hz}, 1 \mathrm{H}, \mathrm{H}-8), 8.19$ (d, J = 7.2 Hz, 2H, H-3' \& H$\left.5^{\prime}\right), 7.84(\mathrm{t}, J=8.8 \mathrm{~Hz}, 1 \mathrm{H}, \mathrm{H}-7), 7.70(\mathrm{t}, J=8.4$ $\mathrm{Hz}, 1 \mathrm{H}, \mathrm{H}-6), 7.61$ (d, J = 7.2 Hz, 1H, H-5), 7.49 (d, J = 8.4 Hz, 1H, H-4), $7.34(\mathrm{t}, J=8.0 \mathrm{~Hz}, 1 \mathrm{H}$, $\mathrm{H}-3), 7.04$ (d, J = 7.2 Hz, 2H, H-2' \& H-6'), 6.96 (d, J = 7.6 Hz, 1H, H-2), 4.48-4.32 (m, 1H, H-1"), 2.20 (s, $\left.3 \mathrm{H},-\mathrm{COCH}_{3}\right), 1.57-1.54(\mathrm{~m}, 2 \mathrm{H}, \mathrm{H}-2 ")$, 1.01 (d, $\left.J=6.8 \mathrm{~Hz}, 3 \mathrm{H}, \mathrm{CH}_{3}-4 "\right), 0.85$ (t, $J=7.2$ $\left.\mathrm{Hz}, 3 \mathrm{H}, \mathrm{CH}_{3}-3 "\right) ;$ El-MS (m/z): 396 (8\%), 275 (4 \%), $198(30 \%), 134$ (9\%), 127 (100\%), 102 (3 $\%), 65(41 \%), 57$ (4\%), 51 (5\%).

\section{N-(Pentan-1-yl)-N-(naphthalen-1-yl)-4- acetamidobenzenesulfonamide $(5 \mathrm{~g})$}

Purple Crystalline Solid; Yield: 75 \%; M.P: 230 ${ }^{\circ} \mathrm{C}$; Molecular Formula: $\mathrm{C}_{23} \mathrm{H}_{26} \mathrm{~N}_{2} \mathrm{O}_{3} \mathrm{~S}$; Molecular Mass: 410; IR ( $\left.\mathrm{KBr}, \mathrm{cm}^{-1}\right) v_{\text {max }}: 3053$ (Ar C-H), 1699 ( $\mathrm{Ar} \mathrm{C}=\mathrm{C}), 1676$ (-HNC=O), $1398\left(\mathrm{SO}_{2}\right)^{-}$; ${ }^{1} \mathrm{H}-\mathrm{NMR}\left(\mathrm{CDCl}_{3}, 400 \mathrm{MHz}, \delta / \mathrm{ppm}\right): 8.19(\mathrm{~d}, \mathrm{~J}=$ $\left.7.6 \mathrm{~Hz}, 2 \mathrm{H}, \mathrm{H}-3^{\prime} \& \mathrm{H}-5^{\prime}\right), 7.83$ (dt, $J=8.4,5.6 \mathrm{~Hz}$, $1 \mathrm{H}, \mathrm{H}-6), 7.71(\mathrm{~d}, \mathrm{~J}=8.4 \mathrm{~Hz}, 1 \mathrm{H}, \mathrm{H}-4), 7.51-7.48$ (m, 2H, H-7 \& H-8), 7.32 (t, J = 8.0 Hz, 1H, H-3), 7.24 (d, $J=7.2 \mathrm{~Hz}, 1 \mathrm{H}, \mathrm{H}-2), 6.86(\mathrm{~d}, J=7.2 \mathrm{~Hz}$, $2 \mathrm{H}, \mathrm{H}-2$ ' \& H-6'), 3.69 (t, J = $\left.8.0 \mathrm{~Hz}, 2 \mathrm{H}, \mathrm{H}-1^{\prime \prime}\right)$, 3.41-3.35 (m, 2H, H-2"), $2.17\left(\mathrm{~s}, 3 \mathrm{H},-\mathrm{COCH}_{3}\right)$, 1.23-1.17 (m, 4H, H-3", H-4"), 0.87 (t, J = 7.2 Hz, $3 \mathrm{H}, \mathrm{CH}_{3}-5$ "); EI-MS (m/z): 410 (2\%), 275 (11\%), 
198 (14\%), 134 (7 \%), 127 (100\%), 102 (3\%), $71(7 \%), 65$ (47\%), 51 (7\%).

\section{$N$-(Pentan-2-yl)-N-(naphthalen-1-yl)-4- acetamidobenzenesulfonamide $(5 \mathrm{~h})$}

Black Sticky Solid; Yield: $68 \%$; Molecular Formula: $\mathrm{C}_{23} \mathrm{H}_{26} \mathrm{~N}_{2} \mathrm{O}_{3} \mathrm{~S}$; Molecular Mass: 410; IR $\left(\mathrm{KBr}, \mathrm{cm}^{-1}\right) v_{\max }: 3025$ (Ar C-H), 1672 (Ar C=C), 1657 (-HNC=O), $1396\left(\mathrm{SO}_{2-}\right) ;{ }^{1} \mathrm{H}-\mathrm{NMR}\left(\mathrm{CDCl}_{3}\right.$, $400 \mathrm{MHz}, \delta / \mathrm{ppm}): 8.27$ (d, J = $8.4 \mathrm{~Hz}, 1 \mathrm{H}, \mathrm{H}-8$ ), $8.19\left(\mathrm{~d}, J=7.2 \mathrm{~Hz}, 2 \mathrm{H}, \mathrm{H}-3^{\prime} \& \mathrm{H}-5^{\prime}\right), 7.85(\mathrm{t}, J=$ $8.8 \mathrm{~Hz}, 1 \mathrm{H}, \mathrm{H}-6), 7.70$ (dd, $J=6.4,2.4 \mathrm{~Hz}, 1 \mathrm{H}$, $\mathrm{H}-5), 7.52-7.46$ (m, 2H, H-4 \& H-7), 7.35-7.29 (m, $1 \mathrm{H}, \mathrm{H}-3), 7.05$ (d, J = 7.2 Hz, 2H, H-2' \& H-6'), $6.95(\mathrm{~d}, J=7.2 \mathrm{~Hz}, 1 \mathrm{H}, \mathrm{H}-2), 4.58-4.42(\mathrm{~m}, 1 \mathrm{H}$, $\mathrm{H}-1 "), 2.20\left(\mathrm{~s}, 3 \mathrm{H},-\mathrm{COCH}_{3}\right), 1.80-1.40(\mathrm{~m}, 2 \mathrm{H}$, $\mathrm{H}-2 "), 1.40-1.12\left(\mathrm{~m}, 2 \mathrm{H}, \mathrm{H}-3^{\prime \prime}\right), 1.11$ (t, $J=6.4$ $\left.\mathrm{Hz}, 3 \mathrm{H}, \mathrm{CH}_{3}-5 "\right), 0.81$ (t, $\left.J=7.2 \mathrm{~Hz}, 3 \mathrm{H}, \mathrm{CH}_{3}-4 "\right)$; El-MS (m/z): 410 (5\%), 275 (10\%), 198 (17\%), $134(14 \%), 127$ (100\%), $102(4 \%), 71(6 \%), 65$ (35\%), 51 (6\%).

\section{$\mathbf{N}$-(Heptan-1-yl)-N-(naphthalen-1-yl)-4- acetamidobenzenesulfonamide (5i)}

Purple Sticky Solid; Yield: $74 \%$; Molecular Formula: $\mathrm{C}_{25} \mathrm{H}_{30} \mathrm{~N}_{2} \mathrm{O}_{3} \mathrm{~S}$; Molecular Mass: 438; IR $\left(\mathrm{KBr}, \mathrm{cm}^{-1}\right) v_{\text {max }}: 3039$ (Ar C-H), 1648 (Ar C=C), 1645 (-HNC=O), $1398\left(\mathrm{SO}_{2-}\right) ;{ }^{1} \mathrm{H}-\mathrm{NMR}\left(\mathrm{CDCl}_{3}\right.$, $400 \mathrm{MHz}, \delta / \mathrm{ppm}$ ): 8.19 (d, J=7.6 Hz, 2H, H-3' \& H-5'), 7.83-7.79 (m, 2H, H-5 \& H-6), 7.51 (d, $J=$ $7.6 \mathrm{~Hz}, 1 \mathrm{H}, \mathrm{H}-8), 7.50(\mathrm{t}, J=5.2 \mathrm{~Hz}, 1 \mathrm{H}, \mathrm{H}-7)$, $7.48(\mathrm{~d}, J=7.6 \mathrm{~Hz}, 1 \mathrm{H}, \mathrm{H}-4), 7.32-7.28(\mathrm{~m}, 2 \mathrm{H}$, $\mathrm{H}-2$ \& $\mathrm{H}-3), 6.86$ (d, J = 7.2 Hz, 2H, H-2' \& H-6'), 3.42-3.35 (m, 2H, H-1"), 2.19 (s, 3H, $-\mathrm{COCH}_{3}$ ), 1.30-1.10 (m, 10H, H-2" to H-6"), 0.80 (t, $J=7.2$ $\mathrm{Hz}, 3 \mathrm{H}, \mathrm{CH}_{3}-7$ "); El-MS (m/z): 438 (1\%), 275 (3 \%), 198 (12\%), 134 (8\%), 127 (100\%), 102 (2 \%), 99 (8 \%), 65 (32\%), 51 (4\%).

\section{$N$-Benzyl-N-(naphthalen-1-yl)-4- acetamidobenzenesulfonamide (5j)}

Move Amorphous Solid; Yield: 76 \%; M.P: 180 ${ }^{\circ} \mathrm{C}$; Molecular Formula: $\mathrm{C}_{25} \mathrm{H}_{22} \mathrm{~N}_{2} \mathrm{O}_{3} \mathrm{~S}$; Molecular Mass: 430; IR ( $\left.\mathrm{KBr}, \mathrm{cm}^{-1}\right) v_{\max }: 3280$ (Ar C-H), 1639 ( $\mathrm{Ar} \mathrm{C}=\mathrm{C}), 1601$ (-HNC=O), $1370\left(\mathrm{SO}_{2-}\right)$; ${ }^{1} \mathrm{H}-\mathrm{NMR}\left(\mathrm{CDCl}_{3}, 400 \mathrm{MHz}, \delta / \mathrm{ppm}\right): 7.92(\mathrm{~d}, J=$ $8.4 \mathrm{~Hz}, 1 \mathrm{H}, \mathrm{H}-8), 7.73(\mathrm{~d}, J=8.8 \mathrm{~Hz}, 2 \mathrm{H}, \mathrm{H}-3$ ' \& H-5'), 7.65-7.55 (m, 2H, H-5 \& H-6), 7.38 (d, J = $8.4 \mathrm{~Hz}, 1 \mathrm{H}, \mathrm{H}-4), 7.36(\mathrm{t}, J=8.0 \mathrm{~Hz}, 1 \mathrm{H}, \mathrm{H}-7)$, 7.29-7.22 (m, 2H, H-3 \& H-2), 7.15-7.09 (m, 5H, $\mathrm{H}-2$ " to H-6"), 6.85 (d, J = 7.2 Hz, 2H, H-2' \& H6'), 2.87 (s, 2H, H-7"), 2.17 (s, 3H, $-\mathrm{COCH}_{3}$ ); ElMS (m/z): 430 (4\%), 275 (6\%), 198 (16\%), 134 (13\%), 127 (44\%), 102 (4\%), 91 (100\%), 65 (47\%), 51 (3\%).
N-(2-Chlorobenzyl)-N-(naphthalen-1-yl)-4acetamidobenzenesulfonamide (5k)

Move Amorphous Solid; Yield: 68 \%; M.P: 160 ${ }^{\circ} \mathrm{C}$; $\quad$ Molecular Formula: $\mathrm{C}_{25} \mathrm{H}_{21} \mathrm{~N}_{2} \mathrm{O}_{3} \mathrm{SCl}$; Molecular Mass: 464; IR ( $\left.\mathrm{KBr}, \mathrm{cm}^{-1}\right) v_{\max }: 3019$ (Ar C-H), 1673 (Ar C=C), 1657 (-HNC=O), 1382 $\left(\mathrm{SO}_{2}-\right), 765(\mathrm{C}-\mathrm{Cl}) ;{ }^{1} \mathrm{H}-\mathrm{NMR}\left(\mathrm{CDCl}_{3}, 400 \mathrm{MHz}\right.$, $\delta / p p m): 8.98$ (d, $J=7.6 \mathrm{~Hz}, 1 \mathrm{H}, \mathrm{H}-8), 7.84(\mathrm{~d}, J=$ 8.0 Hz, 2H, H-3' \& H-5'), 7.73-7.71 (m, 2H, H-5 \& $\mathrm{H}-6), 7.41-7.33$ (m, 2H, H-4 \& H-7), 7.11-7.07 (m, $1 \mathrm{H}, \mathrm{H}-3), 7.05-6.99$ (m, 4H, H-3" to H-6"), 6.95 (d, J = 7.2 Hz, 1H, H-2), 6.91 (d, J = 8.0 Hz, 2H, H-2' \& H-6'), 6.95 (d, J=7.2 Hz, 1H, H-2), 4.86 (s, 2H, H-7"), 2.20 (s, 3H, -COCH ${ }_{3}$; $\operatorname{El}-\mathrm{MS}(\mathrm{m} / \mathrm{z})$ : 466 (2 \%), 464 (6 \%), 275 (7\%), 198 (16\%), 134 (11\%), 127 (37\%), 125 (100\%), 102 (1\%), 98 (23\%), 65 (51\%), 51 (8\%).

\section{N-(4-Chlorobenzyl)-N-(naphthalen-1-yl)-4- acetamidobenzenesulfonamide (5I)}

Purple Crystalline Solid; Yield: 78 \%; M.P: 186 ${ }^{\circ} \mathrm{C}$; $\quad$ Molecular Formula: $\mathrm{C}_{25} \mathrm{H}_{21} \mathrm{~N}_{2} \mathrm{O}_{3} \mathrm{SCl}$; Molecular Mass: 464; IR ( $\left.\mathrm{KBr}, \mathrm{cm}^{-1}\right) v_{\text {max }}$ : 3039 (Ar C-H), 1679 (Ar C=C), 1649 (-HNC=O), 1379 $\left(\mathrm{SO}_{2}-\right), 769(\mathrm{C}-\mathrm{Cl}) ;{ }^{1} \mathrm{H}-\mathrm{NMR}\left(\mathrm{CDCl}_{3}, 400 \mathrm{MHz}\right.$, $\delta / p p m): 7.88$ (d, J = 8.0 Hz, 2H, H-3' \& H-5'), 7.75-7.73 (m, 2H, H-5 \& H-6), $7.63(\mathrm{t}, J=9.2 \mathrm{~Hz}$, $1 \mathrm{H}, \mathrm{H}-8), 7.40-7.36(\mathrm{~m}, 2 \mathrm{H}, \mathrm{H}-4$ \& $\mathrm{H}-7), 7.26(\mathrm{t}, J$ $=10.4 \mathrm{~Hz}, 1 \mathrm{H}, \mathrm{H}-3), 7.13(\mathrm{~d}, J=8.4 \mathrm{~Hz}, 2 \mathrm{H}, \mathrm{H}-3 "$ \& H-5"), 7.05 (d, J = 8.4 Hz, 2H, H-2" \& H-6"), $6.88(\mathrm{~d}, J=7.2 \mathrm{~Hz}, 1 \mathrm{H}, \mathrm{H}-2), 6.84$ (d, J = 7.2 Hz, 2H, H-2' \& H-6'), 4.77 (s, 2H, H-7"), 2.18 (s, 3H, $\left.\mathrm{COCH}_{3}\right)$; El-MS (m/z): 466 (1\%), 464 (3\%), 275 (9\%), 198 (13\%), 134 (10\%), 127 (42\%), 125 (100\%), 102 (2\%), 98 (19\%), 65 (33\%), 51 (6 $\%)$.

\section{N-(4-Bromobenzyl)-N-(naphthalen-1-yl)-4- acetamidobenzenesulfonamide $(5 \mathrm{~m})$}

Move Amorphous Solid; Yield: 80 \%; M.P: 179 ${ }^{\circ} \mathrm{C}$; $\quad$ Molecular Formula: $\mathrm{C}_{25} \mathrm{H}_{21} \mathrm{~N}_{2} \mathrm{O}_{3} \mathrm{SBr}$; Molecular Mass: 509; IR ( $\left.\mathrm{KBr}, \mathrm{cm}^{-1}\right) v_{\max }: 3027$ (Ar C-H), 1679 (Ar C=C), 1657 (-HNC=O), 1398 $\left(\mathrm{SO}_{2}-\right), 680(\mathrm{C}-\mathrm{Br}) ;{ }^{1} \mathrm{H}-\mathrm{NMR}\left(\mathrm{CDCl}_{3}, 400 \mathrm{MHz}\right.$, $\delta / p p m): 7.88$ (d, J = 8.0 Hz, 2H, H-3' \& H-5'), 7.75 (dd, $J=6.8,3.4 \mathrm{~Hz}, 1 \mathrm{H}, \mathrm{H}-8), 7.63-7.58$ (m, $2 \mathrm{H}, \mathrm{H}-5$ \& $\mathrm{H}-6), 7.40-7.36(\mathrm{~m}, 2 \mathrm{H}, \mathrm{H}-4$ \& $\mathrm{H}-7)$, 7.28 (d, $\left.J=8.4 \mathrm{~Hz}, 2 \mathrm{H}, \mathrm{H}-3^{\prime \prime} \& \mathrm{H}-5 "\right), 7.23$ (d, J= $\left.7.6 \mathrm{~Hz}, 2 \mathrm{H}, \mathrm{H}-2{ }^{\prime} \& \mathrm{H}-6^{\prime}\right), 7.01(\mathrm{t}, J=6.8 \mathrm{~Hz}, 1 \mathrm{H}$, $\mathrm{H}-3), 6.99$ (d, J = 8.0 Hz, 2H, H-2" \& H-6"), 6.83 (d, J = 7.2 Hz, 1H, H-2), 2.93 (s, 2H, H-7"), 2.17 (s, 3H, $\left.-\mathrm{COCH}_{3}\right)$; El-MS (m/z): $511(4 \%), 509$ (4 \%), 275 (7\%), 198 (31\%), 170 (100\%), 143 (15 $\%), 134$ (12\%), 127 (35\%), 102 (3\%), 65 (46 $\%), 51(9 \%)$. 
$N$-(2-Phenylethyl)-N-(naphthalen-1-yl)-4acetamidobenzenesulfonamide $(5 n)$

Move Sticky Solid; Yield: $60 \%$; Molecular Formula: $\mathrm{C}_{26} \mathrm{H}_{24} \mathrm{~N}_{2} \mathrm{O}_{3} \mathrm{~S}$; Molecular Mass: 444; IR $\left(\mathrm{KBr}, \mathrm{cm}^{-1}\right) v_{\text {max }}: 3015(\mathrm{Ar} \mathrm{C}-\mathrm{H}), 1680(\mathrm{Ar} \mathrm{C}=\mathrm{C})$, $1657(-\mathrm{HNC}=\mathrm{O}), 1395\left(\mathrm{SO}_{2-}\right) ;{ }^{1} \mathrm{H}-\mathrm{NMR}\left(\mathrm{CDCl}_{3}\right.$, $400 \mathrm{MHz}, \delta / \mathrm{ppm}$ ): 8.11 (d, $J=9.2 \mathrm{~Hz}, 2 \mathrm{H}, \mathrm{H}-3^{\prime}$ \& $\mathrm{H}^{-5}$ ), 7.84 (dd, $\left.J=8.0,7.6 \mathrm{~Hz}, 1 \mathrm{H}, \mathrm{H}-8\right), 7.65-$ $7.56(\mathrm{~m}, 2 \mathrm{H}, \mathrm{H}-5$ \& $\mathrm{H}-6), 7.53-7.46(\mathrm{~m}, 2 \mathrm{H}, \mathrm{H}-4$ \& $\mathrm{H}-7), 7.35$ (t, $J=8.0 \mathrm{~Hz}, 1 \mathrm{H}, \mathrm{H}-3), 7.28-7.12(\mathrm{~m}$, $5 \mathrm{H}, \mathrm{H}-2$ " to $\mathrm{H}-6 "), 7.02\left(\mathrm{~d}, J=6.8 \mathrm{~Hz}, 2 \mathrm{H}, \mathrm{H}-22^{\prime}\right.$ \& $\mathrm{H}-6$ '), $6.91(\mathrm{~d}, J=7.2 \mathrm{~Hz}, 1 \mathrm{H}, \mathrm{H}-2), 4.85$ (t, $J=$ $7.6 \mathrm{~Hz}, 2 \mathrm{H}, \mathrm{H}-8$ "), 2.20 (s, 3H, $\left.-\mathrm{COCH}_{3}\right), 2.02$ (t, $J=7.6 \mathrm{~Hz}, 2 \mathrm{H}, \mathrm{H}-7 ") ;$ El-MS (m/z): $444(1 \%)$, 275 (8\%), 198 (9\%), 134 (9\%), 127 (67\%), 105 (77\%), 102 (4\%), 91 (100\%), 65 (43\%), 51 (4 $\%)$.

\section{$N$-(3-Phenylpropyl)-N-(naphthalen-1-yl)-4- acetamidobenzenesulfonamide (50)}

Move Sticky Solid; Yield: $68 \%$; Molecular Formula: $\mathrm{C}_{27} \mathrm{H}_{26} \mathrm{~N}_{2} \mathrm{O}_{3} \mathrm{~S}$; Molecular Mass: 458; IR $\left(\mathrm{KBr}, \mathrm{cm}^{-1}\right) v_{\max }: 3057(\mathrm{Ar} \mathrm{C}-\mathrm{H}), 1675(\mathrm{Ar} \mathrm{C}=\mathrm{C})$, $1654(-\mathrm{HNC}=\mathrm{O}), 1384\left(\mathrm{SO}_{2-}\right) ;{ }^{1} \mathrm{H}-\mathrm{NMR}\left(\mathrm{CDCl}_{3}\right.$, $400 \mathrm{MHz}, \delta / \mathrm{ppm}$ ): 8.24 (d, $J=8.0 \mathrm{~Hz}, 2 \mathrm{H}, \mathrm{H}-3^{\prime}$ \& $\left.\mathrm{H}-5^{\prime}\right), 8.02(\mathrm{~d}, J=6.4 \mathrm{~Hz}, 1 \mathrm{H}, \mathrm{H}-8), 7.83-7.79(\mathrm{~m}$, $2 \mathrm{H}, \mathrm{H}-5$ \& $\mathrm{H}-6), 7.60(\mathrm{~d}, J=6.8 \mathrm{~Hz}, 1 \mathrm{H}, \mathrm{H}-4)$, $7.52(\mathrm{dt}, J=6.8,1.2 \mathrm{~Hz}, 1 \mathrm{H}, \mathrm{H}-7), 7.31(\mathrm{t}, J=7.6$ $\mathrm{Hz}, 1 \mathrm{H}, \mathrm{H}-3), 7.27-7.07$ (m, 5H, H-2" to H-6"), $6.99\left(\mathrm{~d}, J=6.8 \mathrm{~Hz}, 2 \mathrm{H}, \mathrm{H}-2^{\prime} \& \mathrm{H}-6^{\prime}\right), 6.85(\mathrm{~d}, J=$ $7.2 \mathrm{~Hz}, 1 \mathrm{H}, \mathrm{H}-2), 2.61$ (t, $J=6.0 \mathrm{~Hz}, 2 \mathrm{H}, \mathrm{H}-9 ")$, $2.18\left(\mathrm{~s}, 3 \mathrm{H},-\mathrm{COCH}_{3}\right), 1.80(\mathrm{t}, J=8.0 \mathrm{~Hz}, 2 \mathrm{H}, \mathrm{H}-$ 7"), 0.93-0.89 (m, 2H, H-8"); El-MS (m/z): $458(3$ \%), 275 (7\%), 198 (15\%), 134 (18\%), 127 (56
\%), 119 (39\%), 105 (83\%), 102 (3 \%), 91 (100 $\%), 65$ (36\%), 51 (5\%).

\section{Anti-enzymatic activity}

Among the synthesized molecules, almost all of them remained active against lipoxygenase enzyme relative to baicalein, a reference standard with $\mathrm{IC}_{50}$ value of $22.4 \pm 1.3 \mu$ moles. $\mathrm{N}$ (butan-1-yl)-N-(naphthalen-1-yl)-4-

acetamidobenzenesulfonamide $(\mathbf{5 e})$ and $\mathrm{N}$ benzyl- $N$-(naphthalen-1-yl)-4-

acetamidobenzenesulfonamide $(\mathbf{5 j})$ showed good lipoxygenase activity due to small aliphatic and unsubstituted aralkyl groups and $\mathrm{IC}_{50}$ values of $132.21 \pm 0.73$ and $133.33 \pm 0.87 \mu \mathrm{moles} / \mathrm{L}$, respectively.

The a-glucosidase enzyme activity results for the synthesized derivatives (5a-o) demonstrate that a few were active with excellent inhibitory potential, based on $\mathrm{IC}_{50}$ values (Table 1). Among these molecules, $5 b, 5 c, 5 \mathrm{~h}, 5 \mathrm{j}, 5 \mathrm{~m}$ and 50 were active but $\mathrm{N}$-(propan-1-yl)-N-(naphthalen-1-yl)-4acetamidobenzenesulfonamide (5b), N-benzyl-N(naphthalen-1-yl)-4-acetamidobenzene

sulfonamide (5j) and N-(4-bromobenzyl)-N(naphthalen-1-yl)-4-

acetamidobenzenesulfonamide $(5 \mathrm{~m})$ were good inhibitors with $\mathrm{IC}_{50}$ values of $189.23 \pm 1.52$, $139.27 \pm 1.81$ and $19.41 \pm 0.55 \mu \mathrm{moles} / \mathrm{L}$ respectively, relative to acarbose with $\mathrm{IC}_{50}$ value of $38.25 \pm 0.12 \mu \mathrm{moles} / \mathrm{L}$. The compound, $5 \mathrm{~m}$, showed good activity against both lipoxygenase and a-glucosidase.

Table 1: Enzyme inhibition activity of synthesized molecules

\begin{tabular}{|c|c|c|c|c|}
\hline \multirow[t]{2}{*}{ Compound } & \multicolumn{2}{|c|}{ Lipoxygenase enzyme } & \multicolumn{2}{|c|}{$\alpha$-Glucosidase enzyme } \\
\hline & Inhibition (\%) & $I C_{50}(\mu M)$ & Inhibition (\%) & $I C_{50}(\mu M)$ \\
\hline 3 & $98.02 \pm 0.97$ & $171.93 \pm 0.89$ & $-2.33 \pm 0.12$ & - \\
\hline $5 a$ & $97.13 \pm 0.87$ & $260.24 \pm 0.92$ & $5.48 \pm 0.17$ & - \\
\hline $5 b$ & $92.34 \pm .137$ & $299.24 \pm 0.94$ & $85.31 \pm 3.34$ & $189.23 \pm 1.52$ \\
\hline $5 c$ & $68.12 \pm 1.13$ & $341.6 \pm 0.97$ & $93.42 \pm 3.22$ & $349.21 \pm 1.93$ \\
\hline $5 d$ & $47.13 \pm 0.78$ & - & $57.21 \pm 2.31$ & $>450$ \\
\hline $5 e$ & $99.41 \pm 0.97$ & $132.21 \pm 0.73$ & $33.41 \pm 0.14$ & - \\
\hline $5 f$ & $81.49 \pm 1.34$ & $273.61 \pm 0.89$ & $30.90 \pm 1.7$ & - \\
\hline $5 g$ & $39.60 \pm 0.67$ & - & $-3.01 \pm 3.12$ & - \\
\hline $5 \mathrm{~h}$ & $96.95 \pm 1.63$ & $256.41 \pm 0.76$ & $67.58 \pm 2.64$ & $388.21 \pm 1.27$ \\
\hline $5 i$ & $63.76 \pm 0.69$ & $355.61 \pm 0.68$ & $30.55 \pm 2.34$ & - \\
\hline $5 j$ & $98.51 \pm 1.18$ & $133.33 \pm 0.87$ & $93.11 \pm 2.12$ & $139.27 \pm 1.81$ \\
\hline $5 k$ & $3.23 \pm 0.08$ & - & $2.71 \pm 1.55$ & - \\
\hline 51 & $2.87 \pm 2.15$ & - & $8.45 \pm 1.34$ & - \\
\hline $5 m$ & $95.93 \pm 1.23$ & $139.7 \pm 0.51$ & $83.21 \pm 1.25$ & $19.41 \pm 0.55$ \\
\hline $5 n$ & $8.12 \pm 0.77$ & - & $4.63 \pm 1.22$ & - \\
\hline 50 & $86.93 \pm 0.89$ & $256.6 \pm 0.69$ & $83.21 \pm 3.75$ & $341.33 \pm 2.43$ \\
\hline Control & $\begin{array}{l}93.79 \pm 1.27 \\
\text { (Baicalein) }\end{array}$ & $22.4 \pm 1.3$ & $\begin{array}{l}92.23 \pm 0.14 \\
\text { (Acarbose) }\end{array}$ & $38.25 \pm 0.12$ \\
\hline
\end{tabular}




\section{DISCUSSION}

The sulfonamide (compound 3) was obtained as an amorphous powder. Its molecular formula was determined through HR-MS showing $[\mathrm{M}]^{+}$peak at $\mathrm{m} / \mathrm{z} 340.3962$ corresponding to the molecular formula $\mathrm{C}_{18} \mathrm{H}_{16} \mathrm{~N}_{2} \mathrm{O}_{3} \mathrm{~S}$ (Calcd. for 340.4162 ). The molecular formula is also supported by the protons in its ${ }^{1} \mathrm{H}$-NMR spectrum. The signals in its ${ }^{1} \mathrm{H}$-NMR spectrum, appearing at $\delta 8.24(\mathrm{~d}, \mathrm{~J}=$ $\left.8.4 \mathrm{~Hz}, 2 \mathrm{H}, \mathrm{H}-3^{\prime} \& \mathrm{H}-5^{\prime}\right)$ and $\delta 6.66(\mathrm{~d}, \mathrm{~J}=13.6$ $\mathrm{Hz}, 2 \mathrm{H}, \mathrm{H}-2^{\prime}$ \& $\mathrm{H}-6^{\prime}$ ) are typical for the protons of the p-substituted ring and a singlet at $\delta 2.16(\mathrm{~s}$, $3 \mathrm{H},-\mathrm{COCH}_{3}$ ) for acetamyl group. 1-Naphthyl group showed different signals at $\delta 7.80(\mathrm{~d}, \mathrm{~J}=$ $6.4 \mathrm{~Hz}, 1 \mathrm{H}, \mathrm{H}-4), 7.66$ (dd, J = 6.4, $1.9 \mathrm{~Hz}, 1 \mathrm{H}$, $\mathrm{H}-5), 7.66(\mathrm{dd}, \mathrm{J}=6.4,1.9 \mathrm{~Hz}, 1 \mathrm{H}, \mathrm{H}-8), 7.49(\mathrm{dt}$, $\mathrm{J}=8.4,3.6 \mathrm{~Hz}, 1 \mathrm{H}, \mathrm{H}-6), 7.49(\mathrm{dt}, \mathrm{J}=8.4,3.6$ $\mathrm{Hz}, 1 \mathrm{H}, \mathrm{H}-7), 7.43(\mathrm{t}, \mathrm{J}=8.0 \mathrm{~Hz}, 1 \mathrm{H}, \mathrm{H}-3$ ) and $7.35(\mathrm{~d}, \mathrm{~J}=6.4 \mathrm{~Hz}, 1 \mathrm{H}, \mathrm{H}-2)$. On the basis of the above cumulative evidence, the structure of 3 was assigned as $\mathrm{N}$-(naphthalen-1-yl)-4- acetamidobenzenesulfonamide. The mass fragmentation pattern of N-benzyl-N-(naphthalen1-yl)-4-acetamidobenzenesulfonamide (5j) is outlined in Figure 1. Similarly, the proposed structures of other compounds were corroborated.

The compounds bearing sulfamoyl moiety have previously been synthesized by our group $[12,13]$ and their biological activities have been shown to be related to structural modification in the molecules. The overview of compounds showing activity against both enzymes revealed that molecules bearing small $\mathrm{N}$-substitution like propyl group were better inhibitors because of best fit to active site of enzyme. Also $\mathrm{N}$ substitution by benzyl and 4-bromobenzyl presented better result owing to their ability to generate better $\pi-\pi$ interactions with the active site. Thus the compounds $5 \mathrm{~b}, 5 \mathrm{j}$ and $5 \mathrm{~m}$ remained significantly efficient among the whole series of synthesized molecules.

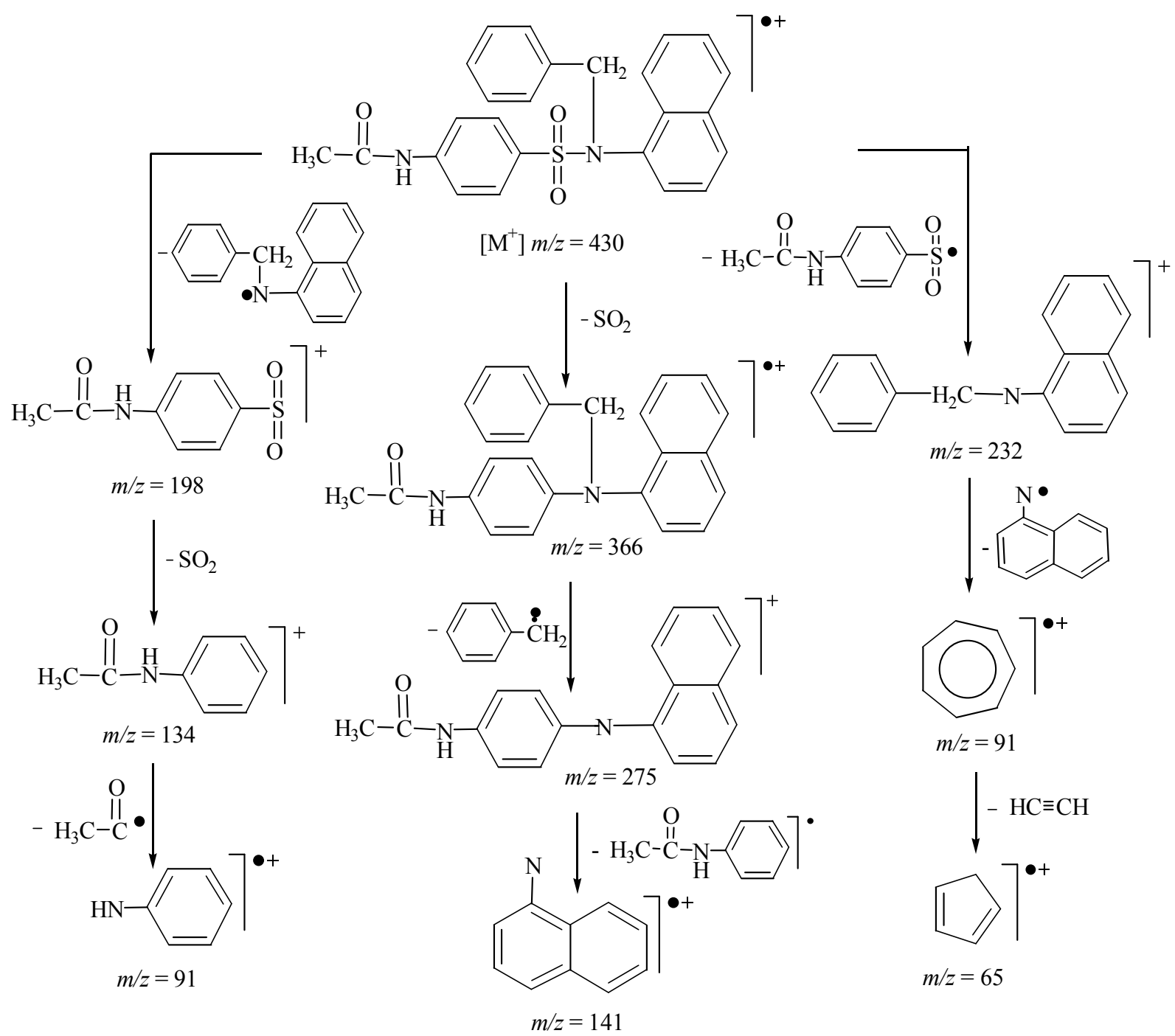

Figure 1: Mass fragmentation pattern of $N$-benzyl- $N$-(naphthalen-1-yl)-4-acetamidobenzene sulfonamide (5j) 


\section{CONCLUSION}

Good yield has been obtained for all the synthesized compounds and definitive spectral structural elucidation attained. The synthesized compounds (5a-0) show potent anti-enzymatic activity against the two enzymes tested, especially lipoxygenase.

\section{REFERENCES}

1. Patrick GL. An Introduction to Medicinal Chemistry, 2nd edn, Oxford University Press, New York, 2001.

2. Vullo D, Franchi M, Gallori E, Antel J, Scozzafava A, Supuran CT. Carbonic anhydrase inhibitors: Inhibition of mitochondrial isozyme $V$ with aromatic and heterocyclic sulfonamides. J Med Chem 2004; 47(5): 1272-1279.

3. Ledford RM, Patel NR, Demenczuk TM, Watanyar A, Herbertz T, Collett MS, Pevear DC. VP1 Sequencing of all human rhinovirus serotype: Insights into Genus Phylogeny and susceptibility to antiviral capsidbinding compounds. J Virol 2004; 78(7): 3663-3674.

4. Lewis AKD, Mok BJ, Tocher DA, Wilden JD, Caddick S. New synthesis of b-Sultams from pentaflourophenyl sulfonates. Org Lett 2006; 8(24): 5513-5515.

5. Roush WR, Cheng JM, Knapp-Reed B, AlvarezHernandez A, McKerrow JH, Hansell E, Engel JC. Potent second generation vinyl sulfonamide inhibitors of the trypanosomal cysteine protease cruzain. Bioorg Med Chem Lett 2001; 11(20): 2759-2762.

6. Kemal C, Louis-Flemberg P, Krupinski-Olsen R, Shorter AL. Reproductive inactivation of soybean lipoxygenase activity. J Biochem 1987; 26: 70647072.

7. Steinhilber D. A target for anti-inflammatory drugs revisited. Curr Med Chem 1999; 6: 71-85.

8. Alitonou GA, Avlessi $F$, Sohounhloue DK, Agnaniet $H$, Bessiere JM, Menut C. Investigations on the essential oil of Cymbopogon giganteus from benin for its potential use as an anti inflammatory agent. Int $\mathrm{J}$ Aromather 2006; 16: 37-41.

9. Chiba S. Molecular mechanism of $\alpha$-glucosiadse and glucoamylase. Biosci Biotech Biochem 1997; 61: 1233-1239.

10. Lebovitz HE. Alpha-glucosidase inhibitors: Endocrinology and Metabolism. Clin North Am 1997; 26: 539-551.

11. Chapdelaine P, Tremblay RR, Dube JY. $\rho$-Nitrophenol- $\alpha$ $D$-Glucopyranoside as substrate for measurement of maltase activity in Human Semen. Clin Chem 1978; 24: 208-211.

12. Abbasi MA, Aziz-ur-Rehman, Muhmood T, Khan KM, Ashraf M, Ejaz SA, Arshad S. Synthesis, characterization and biological screening of various sulfa drugs derived from 2-Anisidine. J Chem Soc Pak 2013; 35(2): 404-410.

13. Abbasi MA, Aziz-ur-Rehman, Qureshi MZ, Khan FM, Khan KM, Ashraf M, Afzal I. 2-Phenitidine derivatives as suitable inhibitors of butyrylcholinesterase. Braz $J$ Pharm Sci 2013; 49(1): 127-133.

14. Abbasi MA, Ahmad S, Aziz-ur-Rehman, Rasool S, Khan $K M$, Ashraf $M$, Nasar $R$, Ismail $T$. Sulfonamide derivatives of 2-amino-1-phenylethane as suitable cholinesterase inhibitors., Trop J Pharm Res 2014; 13(5), 739-745.

15. Baylac S, Racine P. Inhibition of 5-lipoxygenase by essential oils and other natural fragrant extracts. Int $J$ Aromather 2003; 13: 138-142. 